\section{A CASE STUDY ON CHINA'S POLICIES \\ OF PROMOTING WORK RESUMPTION \\ DURING THE COVID-19 PANDEMIC - \\ PERSPECTIVE OF POLICY RESILIENCE}

\author{
Qicheng LU
}

\section{Bin RONG}

\section{Yijia LI}

\author{
Qicheng LU \\ Professor, PhD, Business School of Yunnan University \\ of Finance and Economics, Kunming, Yunnan, China \\ E-mail: luqicheng@aliyun.com
}

Bin RONG (corresponding author)

MA candidate, Business School of Yunnan University of Finance and Economics, Kunming, Yunnan, China Tel.: 0086-199-1694.5502

E-mail: rbrongbin@aliyun.com

\section{Yijia LI}

Associate professor, Information School of Yunnan University of Finance and Economics, Kunming, Yunnan, China E-mail: tilburg@sohu.com

\footnotetext{
* Acknowledgement: Supported by National Natural Science Foundation of China (no. 71872160, no. 71663057, no. 72064043), Key Program of Applied Basic Research Plan in Yunnan Province (no. 2019FA026), Graduate Innovation Fund Program of Yunnan University of Finance and Economics (no. 2021YUFEYC069).
}

DOI: $10.24193 /$ tras.SI2021.4

Published First Online: 12/15/2021

\title{
Abstract
}

During the COVID-19 pandemic, China has achieved high recovery efficiency. One of the most important reasons behind this is the effective policies of promoting work resumption. Why can such policies maintain steady performance despite the high level of environmental uncertainties? This question can be answered from the perspective of policy resilience. This study employed a policy evaluation model for analyzing quantitative data of 342 policies of promoting work resumption. We evaluate the policies through the Policy Modeling Consistency (PMC-index) model and text mining methods. The results show that: first, the contents and elements of all policies have consistent characteristics, including the combination of multiple policy tools, the combination of support for work resumption and pandemic control, the incentives to support effective policy implementation, and the reasonable match between macro and micro policies as well as short-term and long-term policies. Second, among the nine policies that are randomly selected from the sample, one is rated excellent and the other eight are good, indicating that China's policies of promoting work resumption have good resilience.

Keywords: COVID-19 pandemic, policy resilience, China's policies of promoting work resumption, PMC-Index Model. 


\section{Introduction}

Why could China overcome the COVID-19 pandemic peak and achieve rapid economic and social recovery? In recent decades, the losses caused by global crisis events have escalated (Abdulkareem, Elkadi and Breane, 2018, p. 176; Saja et al., 2018, pp. 862-863), and various new risks have emerged one after another. The economic development of a country is inevitably impacted or disturbed by factors such as economic recessions, technological innovation, natural disasters and even terrorism. Thus, the capacity of national crisis management is highly required. In particular, the outbreak of the COVID-19 pandemic has caused serious and lasting impact on the global economy and societies (Milani, 2020, pp. 1-2). It brings serious challenges of economic resilience, especially policy resilience. In 2020, the world's total GDP decreased by $3.3 \%$ year-onyear, while China's GDP increased by $3.1 \%$, which is the only country with an increase of GDP among the top 15 countries. In fact, most of the emerging economies have been seriously troubled by the COVID-19 pandemic, and their economic development has stagnated or even declined. After analyzing the deeper reasons, we found that the Chinese government has designed and issued a series of policies to promote work resumption after the COVID-19 pandemic outbreak, so as to guide enterprises to effectively realize the resumption of work and production, and help enterprises overcome difficulties from the aspects of taxation, epidemic prevention, law, employment and so on. Under the circumstances of limited time and uncertain environment, the policies formulated by the Chinese government have shown strong resilience and effectively promoted the high-quality economic and social recovery. What is the nature of a policy with strong resilience is worth studying by scholars, and it is also an urgent problem to be solved through policy design. This study explores China's policies of promoting work resumption from the perspective of resilience, aiming to promote the development of resilience theory, provide new thinking for policy-making, and have important value for the government to enhance its emergency management ability.

Resilience, also known as elasticity and recovery, refers to the adaptability, recovery and sustainable development ability of various subjects when they encounter external risks, pressures and damages (Walker et al., 2004, p. 2; Alexander, 2013). Resilience has originated from physics and is used to represent the property that an object recovers to its original state after being deformed by external forces (Bozza, Asprone and Manfredi, 2015, p. 1730). In the 1950s, psychology used resilience to denote the ability of individuals or families to actively face and adapt to adversity. In the 1970s, Holling (1973) introduced resilience into system ecology, indicating the ability of the ecosystem to maintain the operation of main functions and structures after being impacted by the outside environment. After that, human beings gradually extended resilience from system ecology to social ecology for the purpose of maximizing social benefits and minimizing environmental impact (Berkes, Folke and Colding, 1998). With VUCA (Volatility, Uncertainty, Complexity and Ambiguity) becoming the pronoun of current environmental characteristics, economic resilience has increasingly become a strategic issue that countries all over the world must pay attention to in the pursuit of 
economic development. It can ensure that economies can make rapid adjustment in the event of impact, and it is the basis for supporting the fine operation of economic and social systems (Martin and Sunley, 2015, p.13). Among them, policy resilience significantly helps to improve economic resilience and is the main driving force for healthy and high-quality development of economy and society after the outbreak of the crisis (Ma, Xiao and Yin, 2018, p. 247; Swanson et al., 2010, p. 924). It refers to the adaptive adjustment ability, reform ability and the vitality stimulated by the policy system when the economic and social system are impacted by the crisis (Capano and Woo, 2017). It resists and solves the consequences of the impact by adjusting policies and reallocating resources, as well as restoring the original economic and social development. For example, after the outbreak of COVID-19 pandemic, more than $20 \%$ of the policies of China's promoting work resumption stimulated the demand for electricity through phased measures such as reducing the price of electricity, subsidies for the use of electricity, and no power failure after arrears. The year-on-year growth rate of power consumption in the second quarter of 2020 increased by $10.4 \%$ compared with the first quarter (People's Daily, 2020), which is regarded as the 'barometer' of economic operation. It reflects the good trend of China's economic recovery (People's Daily Overseas Edition, 2020), and reflects the remarkable effect of the policies of promoting work resumption. Policy design is a complex process, involving multiple actors and multiple interest demands. When facing VUCA situation, this puts forward stricter requirements for policy-making. But the research on policy resilience can provide guidance for the government's effective decision-making.

Policy is an important way to improve economic resilience (Briguglio et al., 2006). However, gaps exist in the current field due to the lack of research on specific policies from the perspective of resilience. The deficiencies are as follows: first, no study has found a suitable perspective and method to analyze the radical advantages of China's policies of promoting work resumption, the possible reasons for this are the limited empirical evidence, lack of policy evaluation tools and inadequate policy resilience theory. Second, as one of the three values of public administration, resilience often contradicts the bureaucratic norms and procedural principles of efficiency. The introduction of resilience theory needs to balance its relationship with other values (Hood, 1991, p. 11). However, the lack of research on the paradigm of policy resilience seriously restricts the consideration of resilience in policy design. Third, policy evaluation mainly focuses on the period or after policy implementation, such as Markov Analysis, Cost Benefit Analysis and Comparison Method. In these two stages of evaluation, policies often waste time and resources because of some inappropriate contents, and these play little role in a highly uncertain environment. Meanwhile, there are few policy evaluation methods before policy implementation, but the evaluation methods have the problem of strong subjectivity, which cannot ensure the objectivity and scientificity of the evaluation results (Yi and Feiock, 2012; Suddaby, 2006; Baniya, Giurco and Kelly, 2021).

In order to solve the limitations of the previous studies, from the perspective of policy resilience, this study analyzes China's policies of promoting work resumption 
by comprehensively using PMC-index model and text mining tools. The difference between this model and the previous policy evaluation model is that it is based on the hypothesis of omnia mobilis as the guiding ideology, the main purpose of this model is that everything in the world is moving and connected. Therefore, any relevant variable should not be ignored or considered unimportant (Ruiz Estrada, Yap and Nagaraj, 2008, p. 188). When selecting variables, the scope of consideration is wider. The analysis system based on this can effectively test the adaptability and stability of policies. Meanwhile, the model adopts the method of text mining, which can avoid the subjectivity of expert scoring and make the policy evaluation more objective and scientific. PMC-index model has been applied to new energy, cultivated land protection, science and technology and other fields for policy evaluation (Yang, Xing and Li, 2020; Kuang et al., 2020; Du, Yuan and Gao, 2019), showing good adaptability and scientificity. The novelty of this study includes three aspects: (1) Taking the COVID-19 pandemic as the research opportunity, this study analyzes the reasons for the steady performance of China's policies of promoting work resumption from the perspective of policy resilience, which responds to recent calls (Dutt, 2016, p. 377). This provides practical value by suggestions on policy making of economic recovery for other countries, especially emerging economies; (2) Policy resilience is mainly reflected in the stability and adaptability of policies in an uncertain environment (Dutt, 2016, p. 375). Guided by these two characteristics, this study applies PMC-index model to analyze policy, which is a new attempt in the field of policy resilience research; (3) Before the implementation of the policy, we can comprehensively analyze its content and characteristics through text mining and quantitative evaluation of the policy text. It can enhance the objective fairness of the evaluation results and put forward scientific and comprehensive policy optimization paths to provide useful enlightenment for improving policy resilience.

\section{Describing the case}

At the beginning of 2020, COVID-19 pandemic broke out all over the world, affecting the normal operation of economy and society. China was also seriously affected by it. The closure of cities began on January 23, and enterprises stopped production in a wide range, resulting in a year-on-year decrease of $6.8 \%$ in China's GDP in the first quarter of 2020. However, it reversed the growth to $3.2 \%$ in the second quarter. China's 'Working arrangements for COVID-19 pandemic prevention and control and economic and social development' conference was released in February 23, 2020, which has made strict arrangements for the next epidemic prevention and control and economic and social development, and has begun the work of promoting work resumption. After that, China's State Council, provinces, municipalities directly under the central government and autonomous regions have successively issued a series of policies on promoting work resumption in a short time, effectively helping enterprises to resume production smoothly and realizing the 'V-shaped' reversal of GDP during the epidemic. During this period, China helped the stagnant economic and social system revitalize through 
policies that aimed at repairing the broken industrial chain, restore transportation, provide financial and resource support, and restore personnel mobility. An example of such as policy is 'Emergency notice on work resumption and dispatching arrangement of key material production enterprises for epidemic prevention and control'. Before the full work resumption, the Chinese government supported the survival of enterprises by maintaining and rebuilding the basic resources needed for enterprise operation. Especially for basic medical protection resources, the government was increasing the production subsidies and reserves, and directly provided them to employees returning to work, so as to prevent and control the epidemic and help enterprises solve the difficulties. In order to solve the major problem of enterprise employees maintaining realistic social distance (Radu, 2021, p. 128), the government issued 'Special action plan for digital empowerment of small and medium-sized enterprises' and other policies. Among these policies, the government has applied new mobile phone applications such as 'Travel Track Card' and 'Health Code' through information technology to scientifically and accurately track epidemic transmission paths. These policies also guide enterprises to realize online office, online financial management, and remote cooperation and so on through digital tools. At the same time, these policies support and guide enterprises to build a supply chain system and sales network through digital operation, which combines online procurement and sales, offline optimal inventory and unmanned distribution, and intelligent logistics. Through a series of policies, the enterprise's ability to deal with sudden crises and the stability of operation have been improved.

Even if there is some structured evidence-based basis in the process of policy design, the implementation effect is not ideal due to the deviation of decision makers' subjective cognition of events or issues, the lack of knowledge, and the change of environment or issues. The governments of most countries have performed poorly in the face of COVID-19 pandemic, but the Chinese government has formulated a series of highly resilient policies for work resumption in a short time. In the face of severe uncertainty in the future, it has played a steady performance and played an important role in economic and social recovery. At the peak of the epidemic in the first half of 2020, China's economy and society achieved a high-quality recovery, which provided a rare opportunity to study policy resilience. Therefore, from the perspective of resilience, this study makes a detailed analysis of the content and characteristics of China's policies of promoting work resumption.

\section{Constructing the analysis model of policy}

This study analyzes policies of promoting work resumption through PMC-index model, which has several advantages. First, it can analyze the internal consistency of policy to reflect the degree of adaptability and stability of policy. Second, the advantages and disadvantages of individual policies can be analyzed. The overall evaluation of policies can be carried out through PMC index, and PMC surface intuitively and 
vividly reflects the advantages and disadvantages of policies from a multi-dimensional perspective in the images, which can be used as a basis to optimize policies and improve the resilience of policies. The application of PMC-index model includes the following four steps: (1) classification of variables and identification of parameters;

(2) establishing multiple input-output tables; (3) measurement of PMC index; and (4) building PMC surfaces.

\subsection{Samples and sorting of policies}

Policies of promoting work resumption studied in this paper come from the official websites of the central government of China and the governments of all provinces and municipalities directly under the central government. The time range of the selection is from January 1, 2020 to June 30, 2020. Irrelevant documents such as meeting minutes and receipts were filtered out, and 342 representative policy documents were selected. These included 7 policy documents issued by the State Council and the central government, 73 policy documents issued by national functional departments, and 262 policy documents issued by regional governments. These 342 documents are sorted out in detail according to policy types (see Table 1).

Table 1: Types of policies for promoting work resumption

\begin{tabular}{cccccc}
\hline Policy Types & Details & Quantity & Proportion & Restraint strength & Operable strength \\
\hline \multirow{3}{*}{ Planning (160) } & Statute & 1 & $0.3 \%$ & Strong & Weak \\
& Plan & 2 & $0.6 \%$ & Medium strong & Weak \\
& Opinion & 52 & $15.2 \%$ & Medium strong & Medium weak \\
& Measure & 105 & $30.7 \%$ & Medium strong & Weak \\
Implementing (182) & Notice & 180 & $52.6 \%$ & Medium weak & Medium strong \\
& Regulation & 2 & $0.6 \%$ & Medium strong & Medium strong \\
\hline Total & & 342 & $100 \%$ & - & - \\
\hline
\end{tabular}

Source: Authors' elaboration

\subsection{Comparative analysis of policy types}

From the perspective of policy implementation, this paper further divides policy types into planning and implementing policies. Planning policy means that it only puts forward the ultimate goal and planning path, and lacks the implementable guidance of policies. The implementing policy elaborates the content of each measure in the policy document, and specifies the specific implementation methods and principles of each measure (Du, Yuan and Gao, 2019, p. 102). By comparing the two main types of policies for resumption of work and production, we found that the implementing policies accounts for $53.2 \%$, which are more operational and restrictive, reflecting the strong intervention of government in promoting work resumption. The government hoped to strengthen the guidance and control of work resumption by policies, so as to promote economic and social recovery. 


\subsection{Variables}

Before establishing the PMC-index model of policy, this paper first needs to determine the relevant variables of the policy. By sorting out 342 policies of promoting work resumption, we use text mining method, ROSTCM6.0 and UCINET tool to extract key words, establish social network and calculate centrality. The detailed steps are shown in Figure 1.

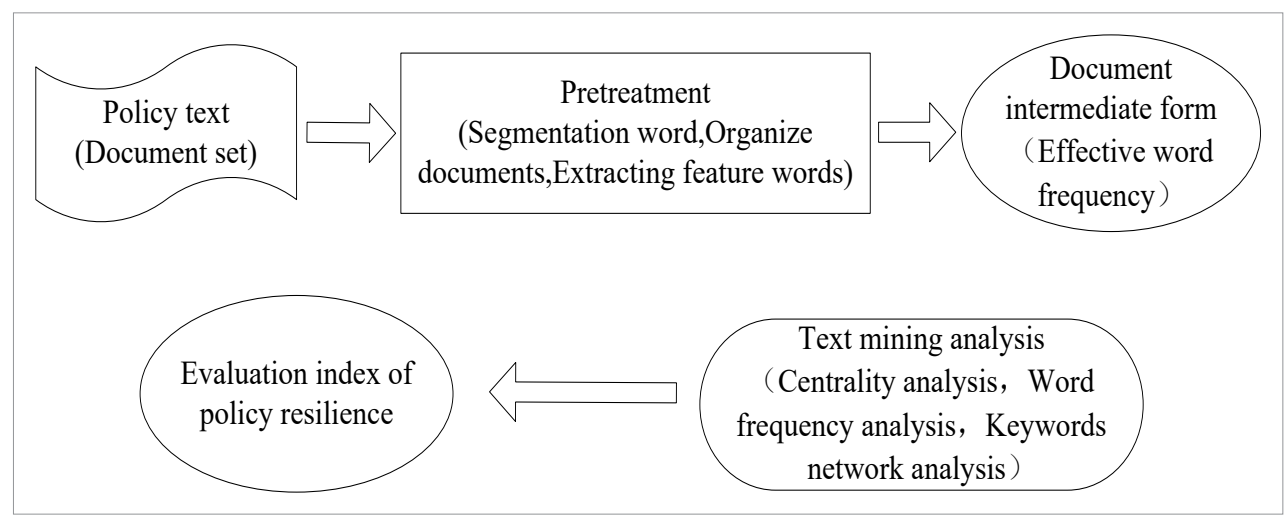

Figure 1: The process of text mining

Source: Authors' elaboration

\subsubsection{Word frequency analysis}

342 policy texts are imported into the text mining database of ROSTCM6.0 software to form a document set for text word segmentation. Further, the word frequency statistics of the document set are carried out, and the results are sorted according to the frequency. Words like 'support', 'development' and 'key' that have no impact on the construction of evaluation indicators but have a high frequency were removed. Afterwards, we summarized effective high-frequency vocabulary. The top 60 words are listed in Table 2 .

Table 2: Effective words and quantities

\begin{tabular}{lccccc}
\hline \multicolumn{1}{c}{ Words } & Quantity & Words & Quantity & Words & Quantity \\
\hline Enterprise & 11791 & SME & 1084 & Start a business & 686 \\
Pandemic & 6529 & Platform & 1032 & Demand & 675 \\
Service & 4887 & Security & 1018 & Guide & 663 \\
Policy & 2546 & Difficulty & 1002 & Science and technology & 642 \\
Work resumption & 2206 & Economics & 1001 & Emergency management & 637 \\
Guarantee & 2184 & Transport & 908 & Hygiene & 618 \\
Obtain employment & 1842 & Technology & 894 & Examine and approve & 605 \\
Staff & 1732 & Consumption & 875 & Human resources & 604 \\
\hline
\end{tabular}




\begin{tabular}{lccccc}
\hline \multicolumn{1}{c}{ Words } & Quantity & Words & Quantity & Words & Quantity \\
\hline Finance & 1706 & Guidance & 852 & Traffic & 601 \\
Project & 1616 & Supervise & 848 & Novel & 586 \\
Loan & 1556 & Healthy & 840 & Supply & 565 \\
Fund & 1518 & Subsidy & 830 & Informationize & 527 \\
Management & 1397 & Agriculture & 801 & Business & 527 \\
\hline Sociology & 1394 & Industry & 779 & Travel & 464 \\
Insurance & 1357 & Apply & 776 & Logistics & 454 \\
\hline Resources & 1224 & Innovate & 766 & Law & 451 \\
Material & 1207 & Mechanism & 746 & Passageway & 432 \\
Bank & 1153 & Pneumonia & 739 & Coronavirus & 421 \\
Financing & 1125 & Medical care & 710 & Graduate & 400 \\
Market & 1108 & Epidemic prevention & 703 & Green & 390 \\
\hline
\end{tabular}

\subsubsection{Keywords network analysis}

In this study, the policy keywords are processed by ROSTCM6.0 software to get the policy keyword network (see Figure 2). The size of the circle in the figure indicates the frequency of keywords in the policies. The length of the line connecting keywords represents the tightness between keywords, reflects the text semantics and forms a social relationship network diagram. The social network diagram of the policy can visually reflect the core structure and radiation degree of the policy and provide a basis for determining the primary and secondary variables of PMC index.

\subsubsection{Centrality analysis}

Through the Net-Degree function in UCINET software, the values of 'Centrality' and 'Network Centralization' of the keyword network of policies of promoting work resumption are obtained, and some effective words required to establish the evaluation system were selected, as shown in Table 3.

Table 3: Some effective words and centrality

\begin{tabular}{lccccc}
\hline \multicolumn{1}{c}{ Words } & Degree & Words & Degree & Words & Degree \\
\hline Enterprise & 46537 & Market & 29029 & Public & 22834 \\
Pandemic & 46422 & Meet an emergency & 28743 & Insurance & 22797 \\
Service & 44115 & Special & 28539 & Logistics & 22594 \\
Guarantee & 43837 & Apply & 28421 & Traffic & 22476 \\
Policy & 42491 & Perfect & 28223 & Hygiene & 22108 \\
Return to work & 37889 & Supervise & 27699 & Obtain employment & 20855 \\
Pneumonia & 37594 & Security & 27258 & Minor enterprises & 20152 \\
Management & 35781 & New type & 27156 & Informationize & 20120 \\
\hline
\end{tabular}




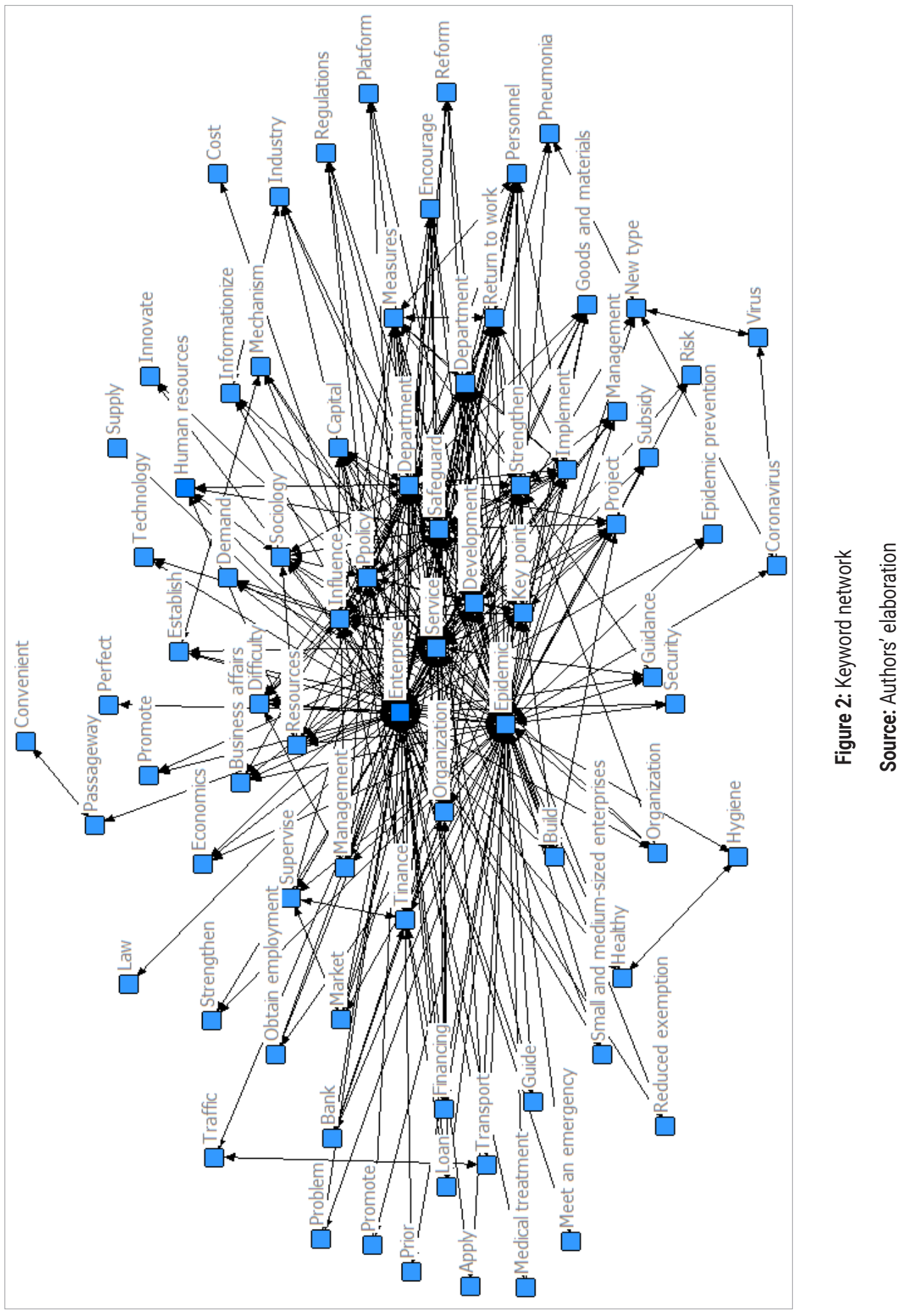


Table 3

\begin{tabular}{lccccc}
\hline \multicolumn{1}{c}{ Words } & Degree & Words & Degree & Words & Degree \\
\hline Capital & 35463 & Epidemic prevention & 26807 & Virus & 20046 \\
Personnel & 34358 & Innovate & 26439 & Coronal & 19945 \\
\hline Platform & 33738 & Technology & 26072 & Industry & 19255 \\
\hline Project & 33359 & Resources & 25942 & Investment & 18873 \\
\hline Material & 33111 & Reduction & 25875 & Start a business & 18636 \\
\hline Mechanism & 32862 & Green & 25401 & System & 18242 \\
Guide & 31871 & Finance & 24728 & Environment & 17174 \\
\hline Healthy & 31468 & Medical care & 24665 & Unemployment & 15991 \\
Demand & 30339 & Examine and approve & 24525 & Support & 15493 \\
Sociology & 29584 & Novel coronavirus & 23490 & Operate & 15124 \\
Transport & 29223 & Economics & 23347 & Staff & 14532 \\
Finance & 29136 & Bank & 22888 & Science & 14248 \\
\hline
\end{tabular}

Note: network centralization $=23.42 \%$

Source: Authors' elaboration

The centrality value can represent the position of a keyword in the whole keyword network. The higher the centrality of a keyword, the higher its position in the network, and the more likely it is to become an indicator of research policy. For example, the word 'pandemic' ranks second in the overall centrality ranking, which is also the background of the research topic, so it is also in a more important position in the keyword table and keyword network diagram.

\subsubsection{Classification of variables and identification of parameters}

Referring to the idea of constructing the index system by Ruiz Estrada (2011, p. 527), and combined with the analysis of high-frequency words and keywords, this paper combines the characteristics of the policies on promoting work resumption with the PMC- index model to construct the PMC evaluation index system. The indicator system includes 10 primary indicators and 50 secondary indicators (see Table 4).

Table 4: Variable setting

\begin{tabular}{|c|c|c|c|c|c|}
\hline NO. & $\begin{array}{l}\text { Primary } \\
\text { variable }\end{array}$ & No. & Secondary variable & No. & Secondary variable \\
\hline \multirow{3}{*}{$\mathrm{X} 1$} & \multirow{3}{*}{$\begin{array}{l}\text { Nature } \\
\text { of policy }\end{array}$} & $\mathrm{X} 1: 1$ & Forecast & $\mathrm{X} 1: 2$ & Supervise \\
\hline & & $\mathrm{X} 1: 3$ & Proposal & $X 1: 4$ & Describe \\
\hline & & X1:5 & Guide & & \\
\hline \multirow{4}{*}{$\mathrm{X} 2$} & \multirow{4}{*}{$\begin{array}{c}\text { Policy } \\
\text { function }\end{array}$} & $X 2: 1$ & Market regulation & $X 2: 2$ & Business assistance \\
\hline & & $X 2: 3$ & Promoting employment & $X 2: 4$ & Encourage entrepreneurship \\
\hline & & $x 2: 5$ & Perfect mechanism & $x 2: 6$ & Strengthen supervision \\
\hline & & $X 2: 7$ & Ensure safety & $X 2: 8$ & Epidemic prevention guidance \\
\hline
\end{tabular}




\begin{tabular}{|c|c|c|c|c|c|}
\hline NO. & $\begin{array}{l}\text { Primary } \\
\text { variable }\end{array}$ & NO. & Secondary variable & NO. & Secondary variable \\
\hline $\mathrm{X} 3$ & $\begin{array}{c}\text { Policy } \\
\text { perspective }\end{array}$ & X3:1 & Macroscopic & X3:2 & Microcosmic \\
\hline \multirow{2}{*}{$\mathrm{X} 4$} & \multirow{2}{*}{$\begin{array}{l}\text { Effective } \\
\text { time }\end{array}$} & $X 4: 1$ & Temporary ( $\mathrm{t} \leq 6$ months) & $X 4: 2$ & Short-term ( 6 months $<\mathrm{t} \otimes 12$ months) \\
\hline & & $X 4: 3$ & Medium-term (12months<t<24months) & $X 4: 4$ & Long-term (24 months<t) \\
\hline \multirow{3}{*}{$\mathrm{X} 5$} & \multirow{3}{*}{$\begin{array}{l}\text { Issuing } \\
\text { agency }\end{array}$} & $X 5: 1$ & State Council and general office & $X 5: 2$ & National ministries and commissions \\
\hline & & $x 5: 3$ & $\begin{array}{l}\text { Provincial and municipal } \\
\text { prefectural party committees }\end{array}$ & $x 5: 4$ & $\begin{array}{l}\text { Provincial and municipal } \\
\text { departments and bureaus }\end{array}$ \\
\hline & & $X 5: 5$ & Other & & \\
\hline \multirow{5}{*}{$x_{6}$} & \multirow{5}{*}{$\begin{array}{l}\text { Policy } \\
\text { field }\end{array}$} & $x 6: 1$ & Economics & $x 6: 2$ & Social services \\
\hline & & $X 6: 3$ & Technology & $\times 6: 4$ & Medical treatment \\
\hline & & $x 6: 5$ & Insurance & $x 6: 6$ & System \\
\hline & & $\times 6: 7$ & Finance & $x 6: 8$ & Industry \\
\hline & & $\times 6: 9$ & Traffic & $X 6: 10$ & Agriculture \\
\hline \multirow{4}{*}{$\mathrm{X} 7$} & \multirow{4}{*}{$\begin{array}{l}\text { Guarantee } \\
\text { incentive }\end{array}$} & $X 7: 1$ & Project support & $X 7: 2$ & Legal guarantee \\
\hline & & $x 7: 3$ & Tax preference & $x 7: 4$ & Resource support \\
\hline & & $X 7: 5$ & Financial support & $X 7: 6$ & Approval optimization \\
\hline & & $\mathrm{X} 7: 7$ & Platform services & & \\
\hline \multirow{2}{*}{$\mathrm{x} 8$} & \multirow{2}{*}{$\begin{array}{l}\text { Policy } \\
\text { system }\end{array}$} & $X 8: 1$ & Include basis & $x 8: 2$ & Include goals \\
\hline & & $x 8: 3$ & Include plans & & \\
\hline \multirow{3}{*}{$x 9$} & \multirow{3}{*}{$\begin{array}{l}\text { Policy } \\
\text { receptor }\end{array}$} & $X 9: 1$ & Country & $X 9: 2$ & Region \\
\hline & & $x 9: 3$ & Industry & $x 9: 4$ & Enterprise \\
\hline & & $x 9: 5$ & Other & & \\
\hline $\mathrm{X} 10$ & $\begin{array}{c}\text { Policy } \\
\text { disclosure }\end{array}$ & & & & \\
\hline
\end{tabular}

Source: Authors' elaboration

\subsubsection{Multiple input-output table}

Multiple input-output table builds a set of optional data analytical framework, which can store a large amount of data and facilitate the statistics and analysis of any single variable. It is composed of ' $m$ ' primary indicators and ' $n$ ' secondary indicators, and the multiple input-output table does not limit the number of indicators. All indicators have no ranking importance and have the same weight (see Table 5). 
Table 5: Multiple input-output table

\begin{tabular}{|c|c|c|c|c|c|c|c|c|c|}
\hline \multicolumn{5}{|c|}{$\mathrm{X} 1$} & \multicolumn{5}{|c|}{$X 2$} \\
\hline \multirow[t]{5}{*}{ X1:1 } & $X 1: 2$ & X1:3 & $\mathrm{X} 1: 4$ & $X 1: 5$ & $X 2: 1$ & $X 2: 2$ & $X 2: 3$ & $X 2: 4$ & $X 2: 5$ \\
\hline & & & & & $X 2: 6$ & $X 2: 7$ & $X 2: 8$ & & \\
\hline & & X3 & & & & & $X 4$ & & \\
\hline & X3:1 & X3:2 & & & $X 4: 1$ & $X 4: 2$ & $X 4: 3$ & $X 4: 4$ & \\
\hline & & $\times 5$ & & & & & $x 6$ & & \\
\hline \multirow[t]{3}{*}{$X 5: 1$} & $X 5: 2$ & $X 5: 3$ & $X 5: 4$ & $X 5: 5$ & $X 6: 1$ & $X 6: 2$ & $X 6: 3$ & $\times 6: 4$ & $\times 6: 5$ \\
\hline & & & & & $X 6: 6$ & $X 6: 7$ & X6:8 & $\times 6: 9$ & $X 6: 10$ \\
\hline & & X7 & & & & & $x 8$ & & \\
\hline$X 7: 1$ & $X 7: 2$ & $X 7: 3$ & $X 7: 4$ & $X 7: 5$ & & $X 8: 1$ & $X 8: 2$ & $X 8: 3$ & \\
\hline \multirow[t]{2}{*}{$X 7: 6$} & $X 7: 7$ & & & & & & & & \\
\hline & & $X 9$ & & & & & $\mathrm{X} 10$ & & \\
\hline X9:1 & X9:2 & X9:3 & $X 9: 4$ & X9:5 & & & $\mathrm{X} 10$ & & \\
\hline
\end{tabular}

Source: Authors' elaboration

\subsection{Calculating the PMC index}

According to the method of Mario Arturo Ruiz Estrada (2011, p. 527), the calculation of PMC index is divided into four steps. First, put the primary and secondary variables into the multiple input-output table. Second, assign values to secondary variables through text mining, formula (1) and formula (2). Third, calculate the score of the primary variables through formula (3). Fourth, summing up the primary index scores of the policies according to formula (4) to obtain PMC index.

$$
\begin{aligned}
& \mathrm{X} \sim \mathrm{N}[0,1] \\
& \mathrm{X}=\{\mathrm{XR}:[0 \sim 1]\} \\
& X_{t}\left(\sum_{j=1}^{n} \frac{X_{i j}}{T\left(X_{i j}\right)}\right) \quad \mathrm{t}=1,2,3,4,5,6, \ldots, \infty
\end{aligned}
$$

In the formula, ' $\mathrm{t}$ ' is the primary variable and ' $\mathrm{j}$ ' is the secondary variable.

$$
P M C=\left[\begin{array}{c}
X 1\left(\sum_{i=1}^{5} \frac{X_{1 i}}{5}\right)+X 2\left(\sum_{j=1}^{8} \frac{X_{2 j}}{8}\right)+X 3\left(\sum_{k=1}^{2} \frac{X_{3 K}}{2}\right) \\
+X 4\left(\sum_{l=1}^{4} \frac{X_{4 l}}{4}\right)+X 5\left(\sum_{m=1}^{5} \frac{X_{5 m}}{5}\right)+X 6\left(\sum_{n=1}^{10} \frac{X_{6 n}}{10}\right) \\
+X 7\left(\sum_{p=1}^{7} \frac{X_{7 p}}{7}\right)+X 8\left(\sum_{q=1}^{3} \frac{X_{8 q}}{3}\right)+X 9\left(\sum_{r=1}^{5} \frac{X_{9 r}}{5}\right)+X 10
\end{array}\right]
$$

Finally, the calculated results are evaluated according to the criteria for evaluating policies (see Table 6). 
Table 6: Criteria for evaluating policies

\begin{tabular}{ccccc}
\hline Score & $9-7$ & $6.99-5$ & $4.99-3$ & $2.99-0$ \\
Criterion & Excellent & Good & Acceptable & Bad \\
\hline
\end{tabular}

\subsection{Building PMC surfaces}

PMC surface is based on PMC index, which can more vividly and intuitively reflect the advantages and disadvantages of policy primary indicators and the consistency level of policies. To build a PMC surface, it is first necessary to generate a PMC matrix. Considering the balance of PMC surface and the symmetry of matrix, X10 is excluded when constructing PMC surface to form a third-order matrix, and PMC surface such as formula (5) is constructed.

$$
\mathrm{PMC} \text { surface }=\left(\begin{array}{lll}
\mathrm{X} 1 & \mathrm{X} 2 & \mathrm{X} 3 \\
\mathrm{X} 4 & \mathrm{X} 5 & \mathrm{X} 6 \\
\mathrm{X} 7 & \mathrm{X} 8 & \mathrm{X} 9
\end{array}\right)
$$

\section{Empirical research}

\subsection{Selecting the evaluation object}

Based on the above analytical framework about policies of promoting work resumption, this study selects 9 policies for evaluation: one item by the State Council and the central government, and two items by national ministries and commissions; one policy issued by Wuhan City, a serious disaster area of COVID-19 pandemic. According to the COVID-19 pandemic cumulative number of confirmed cases, the disaster area is divided into five grades, and a policy is randomly selected in each grade of area. These nine policies are abbreviated as $\mathrm{P} 1$ to $\mathrm{P} 9$ (see Table 9).

\subsection{Calculating PMC index}

In order to make the evaluation results fairly and objectively, the text mining method is used to calculate the secondary variables, which is also better than other evaluation methods. In this study, the nine policies to be evaluated are imported into the text mining database, and the keywords are determined by word segmentation. If there is a keyword corresponding to the name of the secondary variable in the policy text, the secondary variable is assigned 1 , otherwise it is 0 .

According to the assignment of secondary variables, we build a multiple input-output table, further calculate the PMC index, evaluate the policy according to the policy evaluation criteria in Table 6 , and calculate the means of 9 policy primary indicators for horizontal comparison (see Table 10). The depression index is calculated according to PMC index, and they are both presented in the line chart (see Figure 3). 


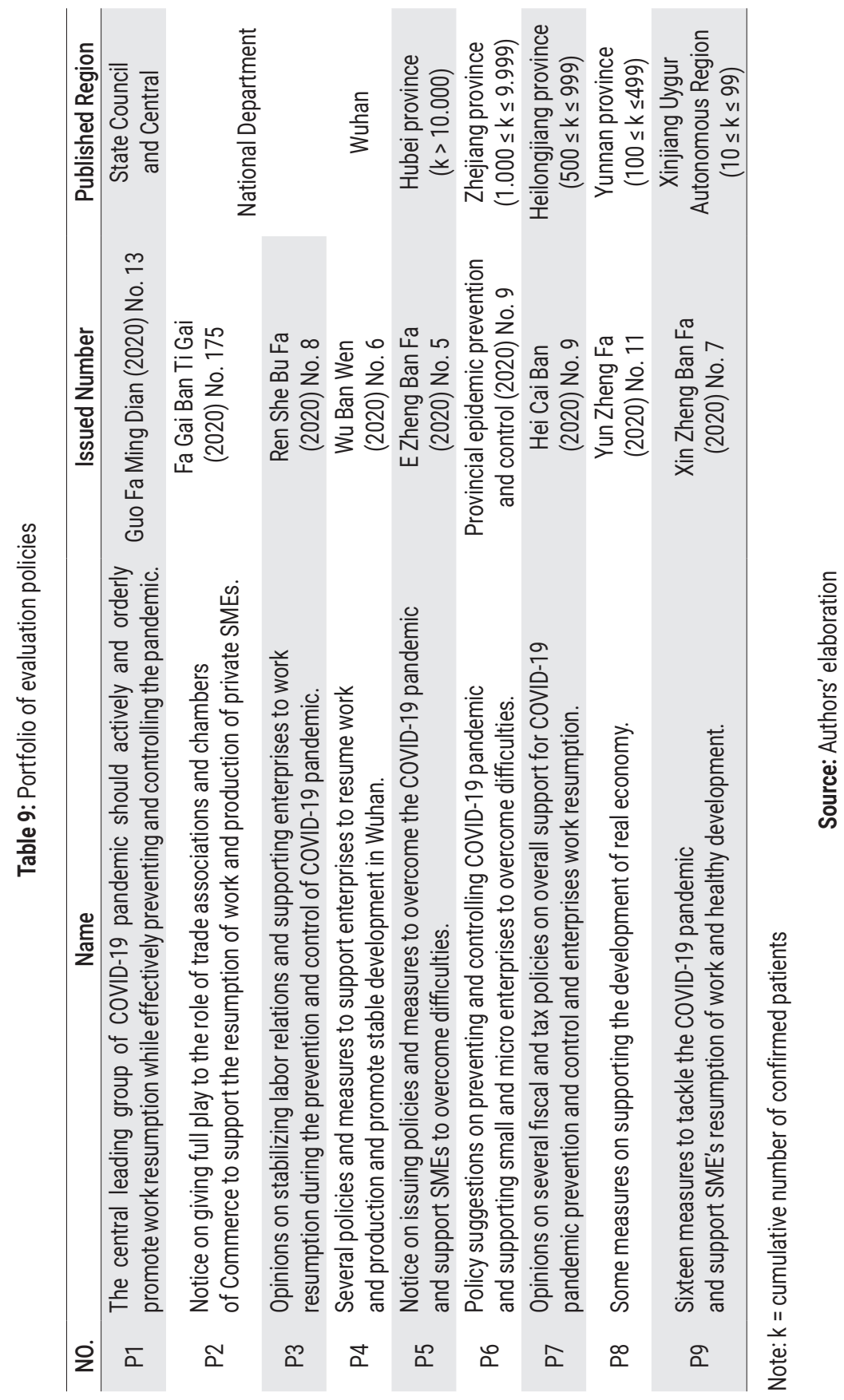


Table 10: PMC index summary

\begin{tabular}{lcccccccccc}
\hline & P1 & P2 & P3 & P4 & P5 & P6 & P7 & P8 & P9 & Mean \\
\hline (X1) Nature of policy & 0.6 & 0.4 & 0.4 & 0.6 & 0.8 & 0.8 & 0.6 & 0.4 & 0.6 & 0.578 \\
(X2) Policy function & 0.625 & 0.875 & 0.5 & 0.875 & 0.75 & 0.5 & 0.25 & 0.625 & 0.875 & 0.653 \\
(X3) Policy perspective & 0.5 & 0.5 & 0.5 & 0.5 & 0.5 & 0.5 & 0.5 & 0.5 & 0.5 & 0.5 \\
(X4) Effective time & 0.5 & 0.5 & 0.5 & 0.75 & 0.5 & 0.25 & 0.25 & 0.5 & 0.5 & 0.472 \\
(X5) Issuing authority & 0.4 & 0.4 & 0.2 & 0.4 & 0.2 & 0.2 & 0.2 & 0.2 & 0.2 & 0.267 \\
(X6) Policy field & 0.4 & 0.7 & 0.5 & 0.8 & 0.5 & 0.4 & 0.6 & 0.8 & 0.8 & 0.611 \\
(X7) Guarantee incentive & 0.429 & 0.714 & 0.571 & 1 & 0.857 & 0.429 & 0.429 & 1 & 0.714 & 0.683 \\
(X8) Policy system & 1 & 1 & 1 & 1 & 0.667 & 0.667 & 0.667 & 0.667 & 0.667 & 0.815 \\
(X9) Policy receptor & 0.6 & 0.8 & 0.8 & 0.8 & 0.6 & 0.8 & 0.8 & 0.8 & 0.6 & 0.733 \\
(X10) Policy disclosure & 1 & 1 & 1 & 1 & 1 & 1 & 1 & 1 & 1 & 1 \\
PMC Index & 6.054 & 6.889 & 5.971 & 7.725 & 6.374 & 5.545 & 5.296 & 6.492 & 6.456 & 6.311 \\
Grade & Good & Good & Good & Excellent & Good & Good & Good & Good & Good & \\
\hline
\end{tabular}

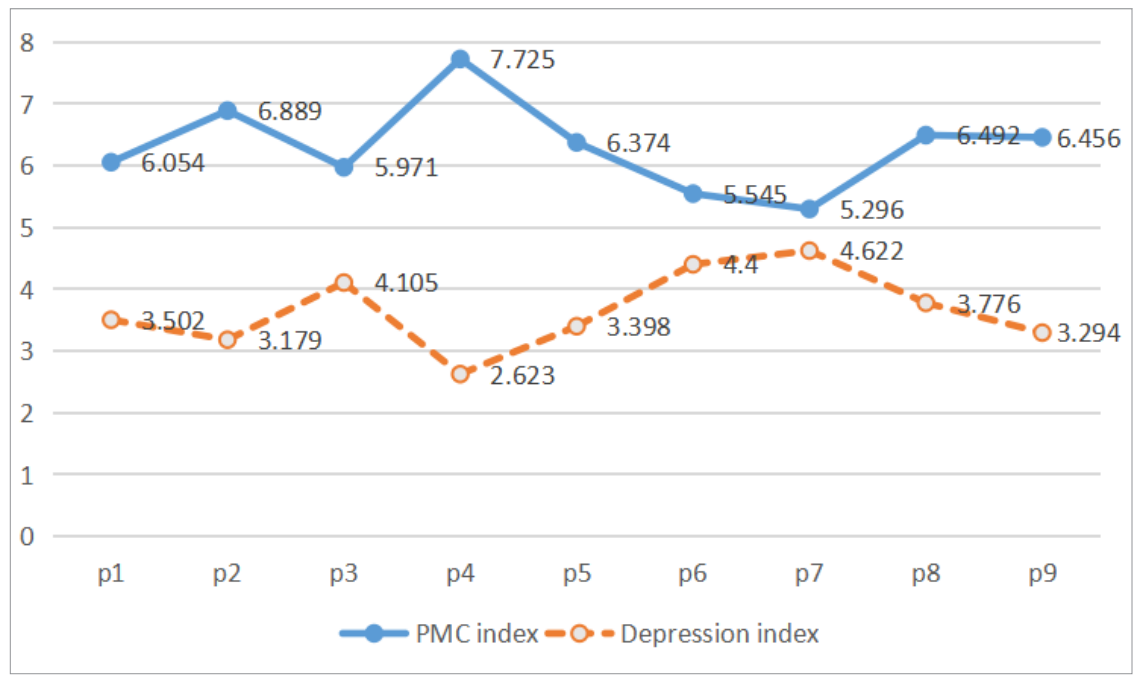

Figure 3: Depression index and PMC index

Source: Authors' elaboration

This paper introduces the policy cobweb graph to compare with PMC surface (see Figure 4). The cobweb graph can intuitively display the scores of policy primary indicators, and can also compare policies horizontally. Although the cobweb graph is not three-dimensional and detailed as PMC surface, it provides another research idea for evaluation policy when only primary indicators need to be analyzed. However, compared with the policy cobweb graph, the surface graph can intuitively reflect the index advantages and disadvantages of a single policy and the depression degree of the policy, which is conducive to the in-depth analysis of a single policy. 


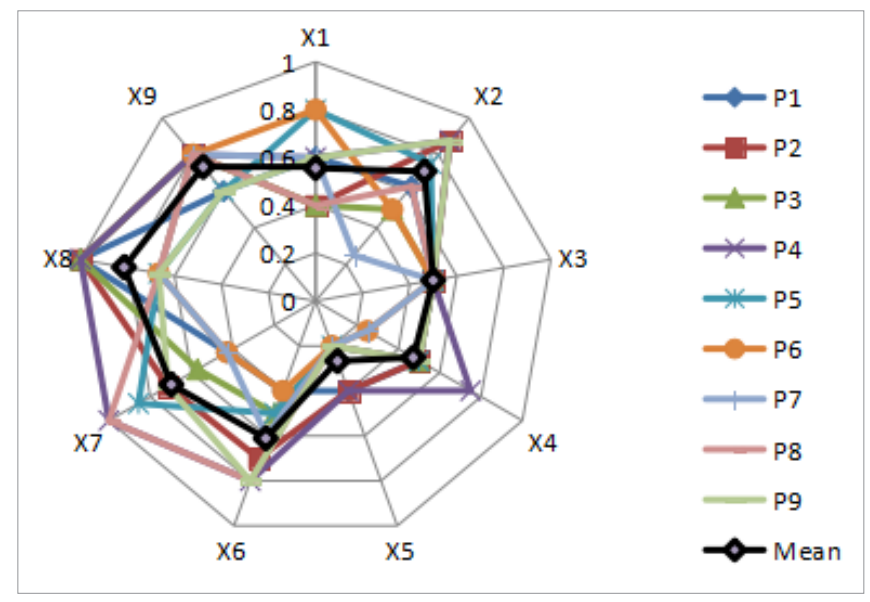

Figure 4: Policy cobweb graph

Source: Authors' elaboration

\subsection{Draw PMC surface graph}

According to the calculation results of PMC index, we gather 9 primary indicators into Matrix of $3 \times 3$, and then draw the PMC surface in the three-dimensional coordinate system according to the corresponding coordinate positions of each primary indicator. This article only shows the PMC surface diagram of policies P4 and P7 (see Figure 5 and Figure 6). On the PMC surface, the areas of each shading represent different values. The concave areas of the surface have relatively low scores, and the convex areas of the surface have relatively high scores.

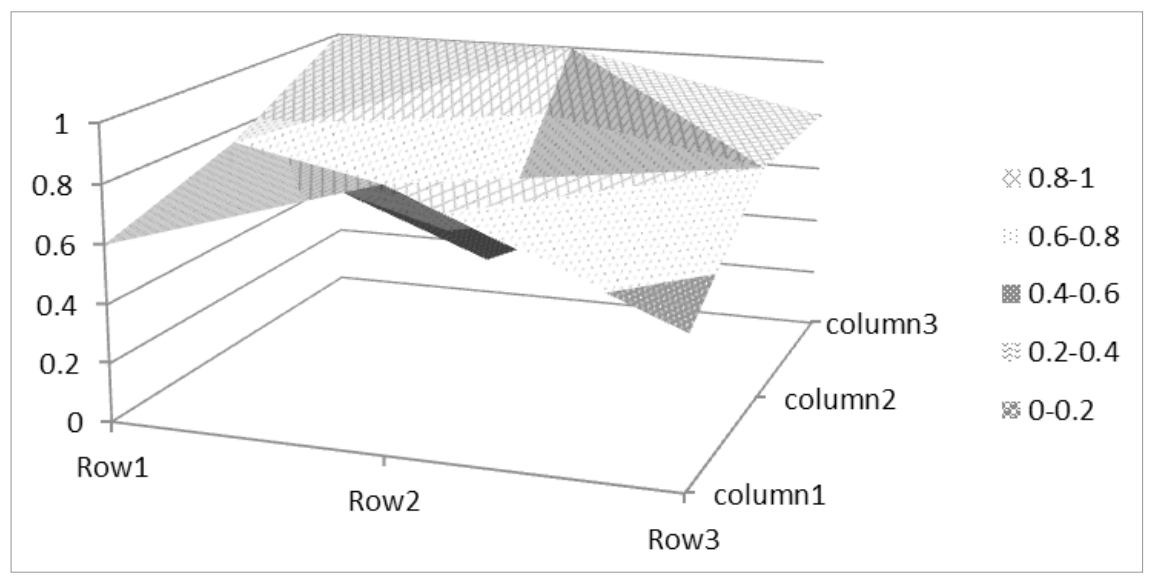

Figure 5: PMC surface of policy 4 


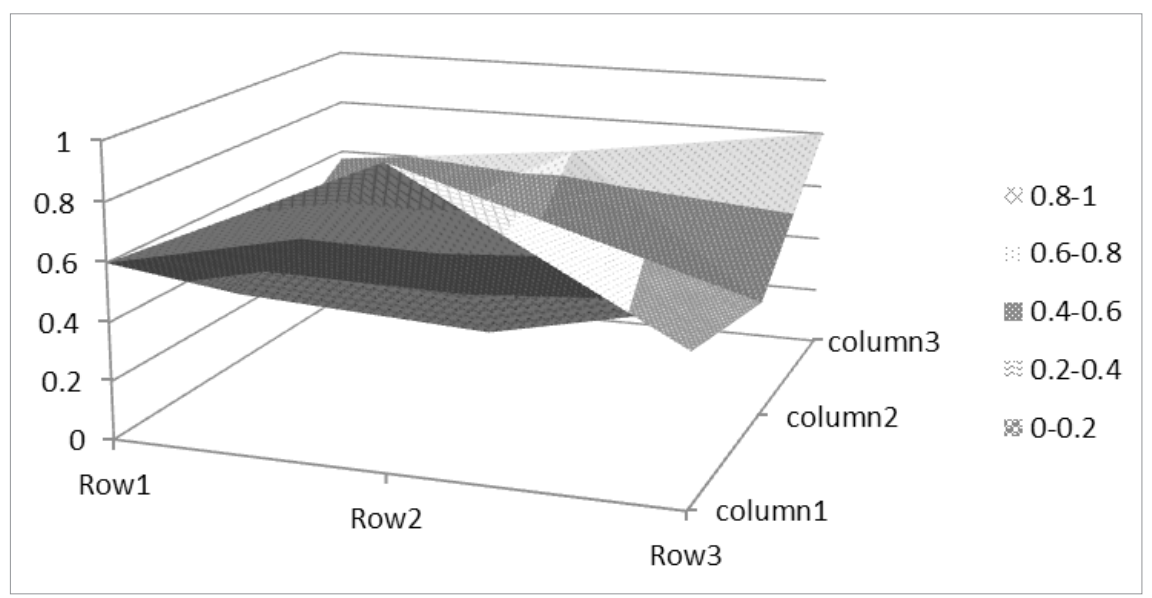

Figure 6: PMC surface of policy 7

\subsection{Analysis of policy evaluation results}

Based on the data results of the above policy analysis, we can analyze and evaluate the policies from two aspects. One is horizontal evaluation, it compares and evaluates various policies, or compares and analyzes the policies to be evaluated with the mean value. The other is vertical evaluation, it analyzes the advantages and disadvantages of individual policies with the help of depression degree and surface graph, and puts forward targeted suggestions.

From the first point of view, the score ranking of $\mathrm{PMC}$ index is $\mathrm{P} 4>\mathrm{P} 2>\mathrm{P} 8>\mathrm{P} 9>$ $\mathrm{P} 5>\mathrm{P} 1>\mathrm{P} 3>\mathrm{P} 6>\mathrm{P} 7$. As the policies of promoting work resumption are emergency management policies, it tends to be temporary and short-term in effective time, so scores are concentrated at 0.5 . In the index policy nature $\mathrm{X} 1$, the scores of policies $\mathrm{P} 1$, P4, P5, P6, P7 and P9 are higher than the average, accounting for $66.7 \%$, indicating that more attention is paid to the form of policy support. As for policy receptor X9, the scores of P2, P3, P4, P6, P7 and P8 are higher than the average level, indicating that the coverage objects of policy regulation are considered comprehensively in this kind of policy formulation, so as to expand the penetration impact of the policy, which is beneficial to ensure the stable effect of the policy in a highly uncertain environment. In the guarantee incentive $\mathrm{X} 7$, the scores of $\mathrm{P} 2, \mathrm{P} 4, \mathrm{P} 5, \mathrm{P} 8$ and $\mathrm{P} 9$ are higher than the average level, indicating the importance of incentive measures for promoting work resumption in policy formulation. In terms of policy function X2, issuing authority X5, policy field X6 and policy system X8, more than $50 \%$ of the policies scored lower than the mean value in these four primary indicators. Therefore, from the overall level of policy evaluation, the policy of promoting work resumption can be improved in four aspects: policy function, issuing authority, policy field and policy system.

Among them, the highest PMC index is P4, with a score of 7.725 as an excellent level policy, which fully shows that the hardest hit areas attach great importance to 
the resumption of work and production. The lowest score of PMC index of P7 among the policies to be evaluated is 5.296, indicating that the resilience of the policy has a certain room for improvement. The scores of policy function X2, effective time X4 and guarantee incentive $\mathrm{X} 7$ are significantly lower than the average level, and the score gap is more than 0.2 . The scores of some primary indicators are also lower than the means to varying degrees, accounting for $60 \%$. In terms of policy function X2, P7 only involves promoting employment and ensuring security. The score of primary index X2 is 0.25 and less than the average score of 0.403 . Therefore, attention should be paid to expanding the scope of policy support. In terms of effective time $\mathrm{X} 4$, it is found that the policy only involves the role within 6 months and has no long-term plan, so it is not specific and forward-looking. In terms of guarantee incentive X7, the policy only involves project support, tax preference and financial support. The guarantee and incentive measures are not comprehensive enough. Policy makers should pay attention to the improvement of this aspect and ensure the effective implementation of policies. Through the analysis of P6 policy, to improve the PMC index, we need to focus on the promotion in the order of X2-X7-X4, but there is a linkage effect between the indicators, so the order is not unique. The specific implementation process should be combined with the specific situation.

According to the depression index of the policy and the PMC surface graph of the policy, we can also evaluate policies vertically. According to Figure 3, the depression index can be divided into three levels: low depression index (0.1-3); acceptable depression index (3.1-4); high depression index (4.1-5). Among them, $\mathrm{P} 4$ is the low depression index, P1, P2, P5, P8 and P9 are the acceptable depression index, and P3, P6 and P7 are the high depression index. As shown in Figure 3, it can be observed that the depression index is inversely proportional to the PMC index. The order of depression degree of the nine policies from strong to weak is P7-P6-P3-P8-P1-P5-P9$\mathrm{P} 2-\mathrm{P} 4$. Among the nine policies, $\mathrm{P} 7$ has the highest depression index. Combined with the PMC surface graph of $\mathrm{P} 7$ in figure 5, $\mathrm{P} 7$ has a high depression in four primary indicators: policy function X2, effective time $\mathrm{X} 4$, issuing authority $\mathrm{X} 5$ and guarantee incentive X7. It can be seen from the policy text that the policy function X2 only involves promoting employment and ensuring safety. Policy makers can consider expanding the function of the policy from market regulation, business assistance, encouraging entrepreneurship, perfect mechanism, strengthening supervision or epidemic prevention guidance, so as to achieve the ultimate goal of helping enterprises with epidemic prevention and normal operation. In terms of X4 effective time, only six months' opinions are involved without long-term deployment. Therefore, some forward-looking opinions and suggestions can be put forward to make the policy play the role of long-term coherence. Since the policy is issued separately by the Heilongjiang Provincial Department of finance, the score of X5 is low. In order to give full play to the linkage effect of the policy, policies could be formulated jointly with other government functional departments. In terms of X7 guarantee incentive, it includes project support, tax preference and financial support. It aims to reduce 
the operating pressure of enterprises by providing financial and tax support. It does not provide incentive and guarantee measures such as law, resources, approval and platform services. The government should comprehensively use incentive measures according to the actual situation to ensure that the policy has strong resilience in the implementation process. Therefore, taking P7 as an example, in order to improve policy resilience, the policy optimization path is $\mathrm{X} 5-\mathrm{X} 2-\mathrm{X} 4-\mathrm{X} 7$, which is different from the first idea. The policy improvement path is not unique and should be determined in combination with the specific situation.

\section{Conclusion and implications}

Based on the analysis of empirical data, several conclusions can be drawn. First, from the perspective of resilience, this paper deeply analyzes the content and element characteristics of China's policies of promoting work resumption. (1) The policies not only considered the industries which were seriously affected by COVID-19 pandemic, but also paid close attention to the stable operation of medical treatment, insurance and other fields in supporting the work resumption. (2) The government has effectively combined various policy tools, using a variety of policy tools such as market regulation, business assistance and encouraging entrepreneurship to promote the resumption of work and production. This also helps to prevent the outbreak of the epidemic again by strengthening supervision and epidemic prevention guidance. The United States issued 'Guidelines for opening up America' on April 26, 2020, focusing on promoting work resumption by regions and stages. However, at this time, the COVID-19 pandemic was not controlled, and there was no scientific protection, strict monitoring and management for the work resumption, resulting in repeated rebound of the COVID-19 pandemic and hindering the work resumption. (3) The government ensures the implementation of policies in terms of law, taxation, funds and services. For example, in order to solve the contradiction between the rework of employees across provinces and the inconsistency of pandemic prevention policies among provinces, the governments of Zhejiang Province and Fujian Province, through consultation, have cooperated to arrange special vehicles to pick up and transport employees in key positions to return to work. Similar to China, Singapore supports the effective implementation of policies by means of employment training, issuing shopping vouchers, increasing community development subsidies and providing one-time financial subsidies for public health prevention clinics. (4) The Chinese government not only makes policies from the macro and micro policy perspectives, but also combines short-term and long-term policies to provide comprehensive guidance for macro strategic planning and specific implementation.

Secondly, the evaluation of 9 randomly selected policies shows that there are $1 \mathrm{ex}-$ cellent grade policy and 8 good grade policies. The results show that China's policies of promoting work resumption have good resilience. (1) The policies of promoting work resumption made by the superior government and the subordinate government exhibit 
excellent consistency, which shows that the subordinate government strongly trusts and supports the superior government. Also, the subordinate government itself has excellent policy implementation ability. (2) The policies of promoting work resumption formulated by different regions have good consistency. In this way, governments can ensure the synchronous recovery of industrial chain and supply chain, and avoid conflicts caused by different recovery processes between regions. This shows that when solving the same problem, the governments of different regions have made high resilience policies through mutual learning and communication. The United States, Italy, Sweden and other countries have highly decentralized their powers in promoting economic and social recovery, while the power of control and coordination has been weakened. Due to the imbalance in the severity of the COVID-19 pandemic, the level of economic development and the speed of work resumption among regions, the conflicts between central and regional governments as well as between different regional governments have been intensified. Therefore, the macro-control policies issued by the state could not be implemented, which damaged the speed and effect of work resumption. Although China has many levels of government management, it implements unified dispatching by the central government, and has set up joint prevention and control mechanisms at all levels to promote cross sectoral cooperation. South Korea and Singapore have adopted similar approaches to ensure strong policy resilience. South Korea has established a central disaster and security strategy headquarters led by the prime minister. They set up local disaster and security strategy headquarters in each region, which are mainly responsible for the local government. Singapore has set up an interdepartmental working group composed of eight government departments, with the deputy prime minister as the adviser and the ministers of health and National Development co-chairing the work. These cases show that the state's unified leadership and rapid decision-making, and the regional governments' mutual coordination and rapid action can help the national and regional governments make and implement strong resilient policies which promote very efficient resumption of work and production.

The contribution of the research mainly includes three aspects. Firstly, from the perspective of resilience, this study deeply studies the content and characteristics of China's policies of promoting work resumption during the COVID-19 pandemic. Secondly, this study introduces the PMC-index model to analyze policies of promoting work resumption from the perspective of resilience for the first time, so as to provide reference for future policy evaluation and resilience related research. Finally, this study puts forward a new perspective and method for the analysis of policies of work resumption. Before the implementation of the policy, through text mining and quantitative analysis, it can save time and resources to the greatest extent, and make the evaluation results objective, scientific and traceable. Therefore, targeted, specific and objective suggestions are put forward to provide reference for policy makers to formulate strong resilient policies in a short time and highly uncertain environment.

Based on the research results and comprehensive analysis, this paper provides practical implications. First, after the impact of the crisis, the market regulation mechanism 
is slow and often needs government intervention. Governments should fully realize the important role of policy resilience in economic health, high-quality and sustainable development, and strengthen the resilience thinking of policy design. Effective benign interaction mechanisms should be established between national and regional governments, between regions seriously and lightly affected by the epidemic, and between regions with good and poor economic and social recovery. According to the characteristics of different tasks, the national system should shift or delegate the decision-making power upward, and establish a coordination mechanism for resumption of work and production between governments at different levels. The state guides and supervises regional policies, and the region builds trust in the state and gives timely feedback. The governments further evaluate and revise policies according to the implementation effect of policies and regional feedback. Avoiding conflict, mutual learning and guidance among regional governments can improve the government's response speed, realize the coordinated development of the policies of work resumption, and enhance the policy resilience. Second, the policies issued by a single functional department are one-sided and limited, so multiple departments should be encouraged to cooperate to introduce the policies of work resumption. The severely divided functional departments need to establish institutionalized or procedural arrangements, make full use of the information technology platform, build a real-time interactive mechanism for the exchange and communication of work resumption policies, increase the linkage of policies, and finally form an efficient policy public service system. Third, the government can improve policy resilience from two policy design perspectives: The first perspective is to increase the proportion of implementation policies, avoid the number of planning policies to exceed implementation policies, and ensure the enforceability of policies. The second perspective is the combination of long-term and short-term policies. The long-term policy can clarify the overall direction of efforts to stimulate and promote social recovery and ensure the stability of policy effect. The short-term policy can avoid falling into the trap of path dependence, which helps to continuously learn and improve the policy according to the monitored and feedback resumption process and the effect of policy implementation, so as to shape the flexibility of the policy. Finally, the government can improve policy resilience in the content of policy design. There are structural uncertainties in the resumption of work and high cognitive uncertainties of policy makers. In order to ensure high policy resilience, policymakers need to combine multiple policy tools to match the actual situation of the country or region. At the same time, policy makers should predict the difficulties that employees and enterprises may encounter in the process of work resumption, and formulate corresponding support and incentive measures to ensure that the policies can be effectively implemented.

The policy resilience measurement model constructed in this study takes China's policies of work resumption as a sample, and does not involve other countries and different types of policies. Future research can conduct comparative research on more countries and types of policies. 


\section{References:}

1. Abdulkareem, M., Elkadi, H. and Breane, M., 'From Engineering to Evolutionary, an Overarching Approach in Identifying the Resilience of Urban Design to Flood', 2018, International fournal of Disaster Risk Reduction, vol. 28, no. 1, pp. 176-190.

2. Alexander, D.E., 'Resilience and Disaster Risk Reduction: An Etymological Journey', 2013, Natural Hazards and Earth System Sciences, vol. 13, no. 11, pp. 2707-2716.

3. Baniya, B., Giurco, D. and Kelly, S., 'Changing Policy Paradigms: How are the Climate Change Mitigation-Oriented Policies Evolving in Nepal and Bangladesh?', 2021, Environmental Science \& Policy, vol. 124, no. 1, pp. 423-432.

4. Berkes, F., Folke, C. and Colding, J., Linking Social and Ecological Systems: Management Practices and Social Mechanisms for Building Resilience, Cambridge: Cambridge University Press, 1998.

5. Bozza, A., Asprone, D. and Manfredi, G., 'Developing an Integrated Framework to Quantify Resilience of Urban Systems Against Disasters', 2015, Natural Hazards, vol. 78, no. 3, pp. $1729-1748$.

6. Briguglio, L., Cordina, G., Bugeja, S. and Farrugia, N., 'Conceptualizing and Measuring Economic Resilience', January 2006, [Online] available at https://www.researchgate.net/ publication/229039198_Conceptalizing_and_measuring_economic_resilience, accessed on October 1, 2021.

7. Capano, G. and Woo, J.J., 'Resilience and Robustness in Policy Design: A Critical Appraisal', 2017, Policy Sciences, vol. 50, no. 3, pp. 1-28.

8. Du, D.L., Gao, K. and Yuan, L., 'Evaluation on Beijing-Tianjin-Hebei Science and Technology Innovation Policy in 2010-2017', 2019, Forum on Science and Technology in China, vol. 35, no. 10, pp. 100-109 (in Chinese).

9. Dutt, A., 'Resilience Thinking: Lessons for Public Administration', 2016, Public Administration, vol. 94, no. 2, pp. 364-380.

10. Holling, C.S., 'Resilience and Stability of Ecological Systems', 1973, Annual Review of Ecology and Systematics, vol. 7, no. 4, pp. 1-23.

11. Hood, C., 'A Public Management for All Seasons?', 1991, Public Administration, vol. 69, no. 1, pp. 3-19.

12. Kuang, B., Han, J., Lu, X., Zhang, X. and Fan, X., 'Quantitative Evaluation of China’s Cultivated Land Protection Policies Based on the PMC-Index Model', 2020, Land Use Policy, vol. 99, no. 1, pp. 124-136.

13. Ma, Z., Xiao, L. and Yin, J., 'Toward a Dynamic Model of Organizational Resilience', 2018, Nankai Business Review International, vol. 9, no. 3, pp. 246-263.

14. Martin, R. and Sunley, P., 'On the Notion of Regional Economic Resilience: Conceptualization and Explanation', 2015, Journal of Economic Geography, vol. 15, no. 1, pp. 1-42.

15. Milani, F., 'COVID-19 Outbreak, Social Response, and Early Economic Effects: A Global VAR Analysis of Cross-country Interdependencies', 2020, fournal of Population Economics, vol. 34, no. 2, pp. 1-30.

16. People's Daily Overseas Edition., 'Electricity Consumption has Reached a New Breakthrough and the Momentum of Economic Recovery is Good', Press release, August 26, 2020, [Online] available at http://www.gov.cn/xinwen/2020-08/26/content_5537436.htm, accessed on October 1, 2021. 
17. People's Daily, 'In the First Half of the Year, Power Investment Increased Rapidly, and the Scale of Power Substitution was About 110 Billion kwh', Press release, July 30, 2020, [Online] available at http:/finance.people.com.cn/n1/2020/0730/c1004-31803083.html, accessed on October 1, 2021.

18. Radu, B., 'Trust in Public Institutions and Compliance with Measures against the COVID-19 Pandemic. Case Study on the Metropolitan Area of Cluj, Romania', 2021, Transylvanian Review of Administrative Sciences, no. 63E, pp. 128-150.

19. Ruiz Estrada, M.A, 'Policy Modeling: Definition, Classification and Evaluation', 2011, Journal of Policy Modeling, vol. 33, no. 4, pp. 523-536.

20. Ruiz Estrada, M.A., Yap, S.F. and Nagaraj, J., 'Beyond the Ceteris Paribus Assumption: Modeling Demand and Supply Assuming Omnia Mobilis', 2008, International fournal of Economics Research, vol. 23, no. 2, pp. 185-194.

21. Saja, A.M.A., Teo, M., Goonetilleke, A. and Ziyath, A.M., 'An Inclusive and Adaptive Framework for Measuring Social Resilience to Disasters', 2018, International fournal of Disaster Risk Reduction, vol. 28, no. 1, pp. 862-873.

22. Suddaby, R., 'From the Editors: What Grounded Theory is Not', 2006, Academy of Management Journal, vol. 49, no. 4, pp. 633-642.

23. Swanson, D., Barg, S., Tyler, S. and Venema, H.D., 'Seven Tools for Creating Adaptive Policies’, 2010, Technological Forecasting \& Social Change, vol. 77, no. 6, pp. 924-939.

24. Walker, B., Holling, C.S., Carpenter, S.R. and Kinzig, A., 'Resilience, Adaptability and Transformability in Social-Ecological Systems', 2004, Ecology and Society, vol. 9, no. 2, pp. 1-9.

25. Yang, T., Xing, C. and Li, X., 'Evaluation and Analysis of New-energy Vehicle Industry Policies in the Context of Technical Innovation in China', 2020, Fournal of Cleaner Production, vol. 281, no. 4, pp. 125-126.

26. Yi, H. and Feiock, R.C., 'Policy Tool Interactions and the Adoption of State Renewable Portfolio Standards', 2012, Review of Policy Research, vol. 29, no. 2, pp. 193-206. 